\title{
Correlation between eye and ear symptoms and lack of teeth, bruxism and other parafunctions in a population of 1006 patients in 2003-2008
}

Maciej Michalak, Joanna Wysokińska-Miszczuk, Magdalena Wilczak, Michał Paulo, Andrzej Bożyk, Janusz Borowicz

Department of Prosthodontics, Medical University of Lublin, Poland

Submitted: 10 June 2011

Accepted: 28 October 2011

Arch Med Sci 2012; 8, 1: 104-110

DOI: $10.5114 /$ aoms.2012.27289

Copyright (c) 2011 Termedia \& Banach

\section{Abstract}

Introduction: Parafunctions (harmful habits) play a crucial role in the formation of temporo-mandibular joint (TMJ) dysfunction syndrome with disc displacement. Disorder symptoms in temporo-mandibular joints manifest themselves in the eye and ear but are usually not associated with the dysfunction of temporo-mandibular joints and that might lead to errors in diagnosis. The aim of the study was to examine the influence of missing teeth and parafunctions on the occurrence of ear and eye symptoms in patients treated in the Department of Prosthodontics of the Medical University of Lublin.

Material and methods: The patient group consisted of 753 women and 253 men aged 10 to 82 years who had been treated in the Department of Prosthodontics, Medical University of Lublin in the years 2003-2008 due to various symptoms associated with temporo-mandibular joint dysfunction.

Results: Eye $(24.84 \%, n=785)$ and ear $(33.38 \%, n=785)$ syndromes occur on average more often in patients with parafunctions than without them $(15.98 \%$, $n=219$ and $23.29 \%, n=219$ ). However, only parafunctions involving tooth contact should be taken into consideration when diagnosing eye and ear syndromes. The data presented here show that the number of missing teeth does not have a significant influence on the frequency of occurrence of parafunctions. Parafunctions have become a very important factor in the diagnosis of diseases and pathological symptoms of eye and ear as the rate at which they occur is growing.

Conclusions: The kind of parafunction is very important. Only those involving tooth contact should be taken into consideration when diagnosing eye and ear syndromes.

Key words: bruxism, parafunctions, eye and ear syndromes.

\section{Introduction}

Symptoms manifesting themselves within the organ of vision and hearing belong to a large group of symptoms resulting from dysfunctions in temporo-mandibular joints. These symptoms include ocular pain, filling of eyes with tears, feeling of blasting from the orbit of the eye, eyelid ptosis, visual sensations, inflammation of the iris, decreased visual acuity, ear pain, feeling of blockage, noise or ringing, ear wax retention, and itching $[1,2]$. However, the clinical problem is very often associated neither with these symptoms nor with the disorder of temporo-mandibular joints, and that might lead to a wrong diagnosis. Therefore, it seems extremely impor-

\author{
Corresponding author: \\ Magdalena Wilczak \\ Department \\ of Prosthodontics \\ Medical University of Lublin \\ 7 Karmelicka \\ 20-081 Lublin, Poland \\ Phone/fax: +48 81532-79-30 \\ E-mail: \\ zaklad_protetyki@wp.pl
}


tant to draw attention to this issue, whose frequency is increasing steadily as a result of stress in daily life that leads to parafunctions of temporomandibular joints [2-8].

Parafunctions are stereotypical, aimless, often unconscious, repetitive masticatory movements, that differ in quality and quantity from physiological movements. There are two main groups of parafunctions: those that involve teeth contact, also referred to as bruxism, such as clenching of teeth and gnashing of teeth; and others that do not involve teeth contact, such as nail biting, biting of the lips and buccal mucosa, habitual chewing of gum, biting on various items (e.g. pens, caps, glasses frames, electronic devices, remote controls, etc.) $[3-5,9,10]$.

Parafunctions play a crucial role in the formation of TMJ dysfunctions with disc displacement and mandibular dysfunctions that result in intra-articular disorders of the temporo-mandibular joint. This leads to painful and painless symptoms, including those listed here and related to the organs of vision and hearing $[3,4,11,12]$. Numerous studies have shown that symptoms of dysfunction occur in 40$70 \%$ of children, and in $60-70 \%$ of adolescents and adults, depending on the assumptions made $[4,5$, 13-15]. The most common symptoms include abnormal condylar motions, pathological sounds (clicks and crackles), pain in pressured areas of the temporo-mandibular joint and free mandibular movement $[1,3,4,6,16,17]$.

Bruxism, i.e. parafunction involving tooth contact, is a common and highly destructive parafunction affecting the masticatory motor system. It is usually diagnosed relatively late as abrasion of the hard tissues of the teeth at an advanced stage. Psychoemotional factors are at the core of this disease, along with increased sensitivity of the muscle receptors and the stretch reflex induced involuntarily by masseter muscle contractions. That results in changes in muscle coordination and the movement pattern of the whole system. Consequently, these processes lead to pain in the stomatognathic system. Various authors distinguish two types of bruxism, namely day and night bruxism. Daily bruxism, also called centric, is a powerful process of clenching with significant force. Night bruxism, described as eccentric, is mostly associated with not only gnashing of teeth, but also clenching of teeth in either centric or eccentric occlusion. Two other types are primary and secondary bruxism. Secondary bruxism has been observed in mental disorders, neurological diseases, disorders of sleep and after the implementation of pharmacotherapy. Bruxism can also be classified as iatrogenic or idiopathic, occurring during the day or at night [18-22].

Panek suggested an indicator to help determine the severity of bruxism. The author assigns high and low scores to evaluate the severity of bruxism. Subjective symptoms and symptoms accompanying other diseases receive low scores. High scores are given to objective symptoms associated with morphological damage in the masticatory organ. Panek distinguishes four degrees of severity of bruxism: assumed bruxism, active bruxism with no major damage to hard tissues of teeth, 'gonethrough' bruxism and fixed, active bruxism with abnormal tooth wear [23].

Masticatory muscles are inherent in the biomechanics of the temporo-mandibular joint. According to Dworkin's and Le Resche's classification, musculo-fascial pain belongs to a group of functional disorders, along with the displacement of the disc associated with pathological symptoms. Local disturbances of individual muscles, that take the form of a tight belt, are characteristic for those disorders. So-called 'trigger points', i.e. points of great sensitivity, appear within a muscle. Acute and chronic pain may disclose itself in areas of the body that are related to that point. There are primary and satellite trigger points, as well as active and latent ones. They are activated in stress conditions, trauma, hormonal disorders or inflammation of the tissues. Pain located in the masseter muscle, the pterygoid medial muscle, sterno-cleido-mastoid muscle and digastric muscle may cause pain, the feeling of noise or blockage in the ear. Pain in the points located in the sterno-cleido-mastoid muscle, trapezius muscle and digastric muscle may manifest itself in the orbital area and the eye socket, causing filling of eyes with tears, dryness, congestion or pale conjunctiva of the eye [24-27].

After the age of 30 years, face height measured between the points nasion and gnathion decreases gradually, as a result of tooth loss in the areas of support. The areas of support are the parts of the dental arch in which occlusal contact between opposing teeth during central occlusion occurs. They are crucial for maintaining the bite height and are found in full dentition within the molars and premolars. Reduction of the height of the face in the lower section can also be caused by teeth wear, teeth movement or leaning of the teeth. This adversely affects the functioning of the entire stomatognathic system, including the temporo-mandibular joints and masticatory muscles. The whole system adapts to the morphological changes according to their severity. After empty spaces in support areas appear, the lower and upper jaw come closer. This leads to adverse shift of the head of the jaw and causes incoordination of muscle work. Both changes result in a decrease of face height and parafunctions affect the functioning of the temporo-mandibular joints. This may cause pathological changes such as abrasion of tuberculae and foveae and articular disc displacement [3, 7-9]. 
The aim of the study was to examine the influence of missing teeth and parafunctions on the occurrence of ear and eye symptoms in patients treated in the Department of Prosthodontics of the Medical University of Lublin.

\section{Material and methods}

The study group consisted of 753 women and 253 men (total 1006) aged 10 to 82 years, treated at the Department of Prosthodontics of the Medical University of Lublin in 2003-2008. Our study has a retrospective design. Patients were divided into groups, taking into account the following criteria:

- criterion I - the kind of parafunctions or absence (Table I),

- criterion II - age: under 18, 18-30, 30-60 years and over 60 years (Table II),

- criterion III - number of missing teeth (Table III),

- criterion IV - gender (female, male) (Table IV),

- criterion $\mathrm{V}$ - the existence of symptoms within the organ of vision and hearing (Table $V$ ).

During the study, the following numbers were assessed: the number of people in each age group, the number of missing teeth for men and women separately in each age group, and the number of missing teeth for men and women altogether. The presence of parafunctions involving and not involving tooth contact, and pathological syndromes both in the eye (pain, feeling of blasting, filling of eye with tears) and ear (pain, feeling of noise) were examined. The relationship between the above criteria was also examined.

The statistical analysis of results used Student's ttest, relative risk (RR) and confidence interval $(\mathrm{Cl})$ to evaluate the significance of differences between average values. A relative risk of 1 means there is no difference in risk between the two groups. An RR of $<1$ means the event is less likely to occur in the experimental group than in the control group. An RR of $>1$ means the event is more likely to occur. Confidence interval gives the high and low bounds for an asymmetric confidence interval around the relative risk.

Table I. The number of patients performing parafunctions that involve tooth contact and do not involve tooth contact (criterion I, $n=1006$ )

\begin{tabular}{|cc|}
\hline Involving tooth contact & Not involving tooth contact \\
\hline $619,61.53 \%$ & $583,57.95 \%$ \\
\hline
\end{tabular}

We have the approval of the local ethics committee to carry out the study.

\section{Results}

Women constituted the majority of patients (74.85\%). The average age of women was 29.47 years, of men 29.02 years. The biggest group among women comprised female patients aged 1830 years (331 women, $33.84 \%$ ), and among men the largest group comprised males aged 18-30 years (107 men, 10.94\%). The highest average number of missing teeth (8.00) among men was noted in the age group over 60 years, whereas among women the highest missing teeth ratio (7.75) was noted in the age group 30-60 years. The average missing teeth ratio in women was 5.44 , in men 4.73 . The highest average number of missing teeth (7.31) was in the age group 30-60 years (Figure 1 ).

Eye $(24.84 \%, n=785)$ and ear $(33.38 \%, n=785)$ syndromes occur on average more often in patients with parafunctions than without them $(15.98 \%$, $n=219$ and $23.29 \%, n=219) . \mathrm{RR}=1.11, \mathrm{Cl}=1.04$ $1.19,10$ Pearson's $\chi^{2}: 7.60941, \mathrm{df}=1, p=0.005808$ (for eye) - the event is more likely to occur, $\mathrm{RR}=1.11, \mathrm{Cl}=1.04-1.18$, Pearson's $\chi^{2}$ : 8.12168, $\mathrm{df}=1, p=0.004375$ (for ear) - the event is more likely to occur (Figures 2 and 3).

In the group suffering from teeth contact involving parafunctions, $36.51 \%(n=619)$ showed ear symptoms, whereas in the group of people not manifesting any signs of tooth-contact parafunctions those symptoms were noted in $22.60 \%$ $(n=385)\left(\mathrm{RR}=1.27, \mathrm{Cl}=1.15-1.39,10\right.$ Pearson's $\chi^{2}$ : 21.4146, $\mathrm{df}=1, p=0.000004$ - the event is more likely to occur. In the group of patients suffering from parafunctions not involving teeth contact, $30.87 \%(n=583)$ suffer from pathological symptoms, whereas among those not suffering from this kind of parafunctions, those symptoms are observed among 31.59\% $(n=421) . R R=0.99$, $\mathrm{Cl}=0.88-1.11,10$ Pearson's $\chi^{2}: 058,518, \mathrm{df}=1$, $p=0.808854$ - the event is less likely to occur.

In the group suffering from parafunctions involving teeth contact $26.66 \%(n=619)$ reported eye symptoms, whereas among the patients who are free from them those symptoms were observed in $16.88 \%(n=385)$ of cases. $\mathrm{RR}=1.22, \mathrm{Cl}=1.11-1.35$, Pearson's $\chi^{2}$ : $12.8367, \mathrm{df}=1, p=0.000340$ - the event is more likely to occur. Among the patients suffering from parafunctions not involving teeth

Table II. Age of the patients divided into four groups: under 18, 18-30, 30-60 and over 60 years (criterion II, $n=978$ -28 patients did not state their age)

\begin{tabular}{|c|c|c|c|c|c|c|c|}
\hline \multicolumn{2}{|c|}{$\begin{array}{l}\text { Age under } 18 \text { years } \\
(x<18)\end{array}$} & \multicolumn{2}{|c|}{$\begin{array}{l}\text { Age } 18 \text { to } 30 \text { years } \\
(18 \leq x<30)\end{array}$} & \multicolumn{2}{|c|}{$\begin{array}{l}\text { Age } 30 \text { to } 60 \text { years } \\
(30 \leq x<60)\end{array}$} & \multicolumn{2}{|c|}{$\begin{array}{l}\text { Age } 60 \text { and over years } \\
\qquad(x \geq 60)\end{array}$} \\
\hline Women & Men & Women & Men & Women & Men & Women & Men \\
\hline $120,12.27 \%$ & $47,4.80 \%$ & $331,33.84 \%$ & $107,10.94 \%$ & $260,26.58 \%$ & $86,8.79 \%$ & $20,2.04 \%$ & $7,0.72 \%$ \\
\hline
\end{tabular}


Table III. The number of patients with missing teeth (criterion III, $n=1006$ )

\begin{tabular}{|cc|}
\hline Women & Men \\
\hline $190,18.89 \%$ & $63,6.26 \%$ \\
\hline
\end{tabular}

contact $24.01 \%(n=583)$ reported pathological symptoms, whereas those symptoms were reported only by $21.38 \%(n=421)$ of the patients not suffering from this kind of parafunctions. $R R=1.06$, $\mathrm{Cl}=0.94-1.20$, Pearson's $\chi^{2}: 961,884, \mathrm{df}=1$, $p=0,326715$ - the event is less likely to occur.

Among the patients performing parafunctions that involve teeth contact the ratio at which ear symptoms occur is higher, whereas among people suffering from parafunctions that do not involve tooth contact it stays the same (Figure 4). Performing parafunctions that involve tooth contact has a bigger influence on the eye symptoms. Parafunctions that do not involve tooth contact do not play a role here (Figure 5).

People with missing teeth who show signs of tooth contact parafunctions constitute $61.84 \%$

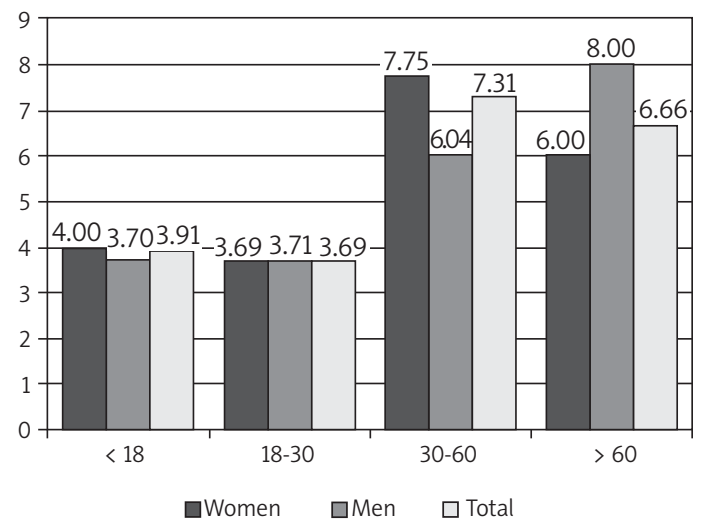

Figure 1. Average number of missing teeth in the examined patients (women and men) in different age groups

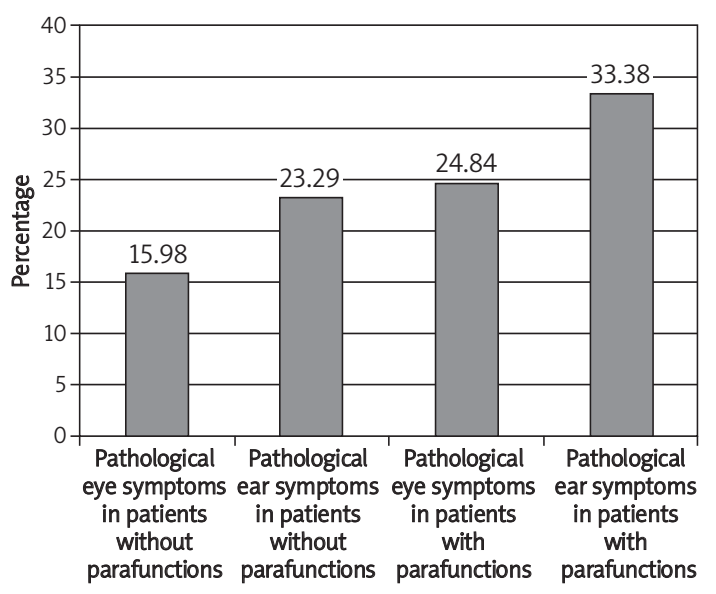

Figure 3. Percentage distribution of eye and ear symptoms among patients who have not engaged parafunctions
Table IV. Patients' gender: female, male (criterion IV, $n=1006$ )

\begin{tabular}{|cc|}
\hline Women & Men \\
\hline $753,74.85 \%$ & $253,25.15 \%$ \\
\hline
\end{tabular}

Table V. The existence of symptoms within the organ

of vision and hearing (criterion $\mathrm{V}, n=1006$ )

\begin{tabular}{|cc|}
\hline \multicolumn{2}{|c|}{ Patients with pathological symptoms } \\
\hline Within the organ of vision & Within the organ of hearing \\
\hline $230,22.86 \%$ & $313,31.11 \%$ \\
\hline
\end{tabular}

( $n=152$ ), whereas those having non-tooth contact parafunctions constitute $62.50 \%(n=152)$. Among people with full dentition, those performing tooth contact parafunctions constitute $61.62 \%(n=852)$ whereas non-tooth contact constitute $57.28 \%$ $(n=852) . R R=1.00, \mathrm{Cl}=0.88-1.15$, Pearson's $\chi^{2}$ : $002,698, \mathrm{df}=1, p=0.958573$ (for people with missing teeth and full dentition who show signs of tooth contact parafunctions) - the event is less likely to occur. $\mathrm{RR}=1.09, \mathrm{Cl}=0.95-1.25$, Pearson's $\chi^{2}$ : $1.44513, \mathrm{df}=1, p=0.229314$ (for people with miss-

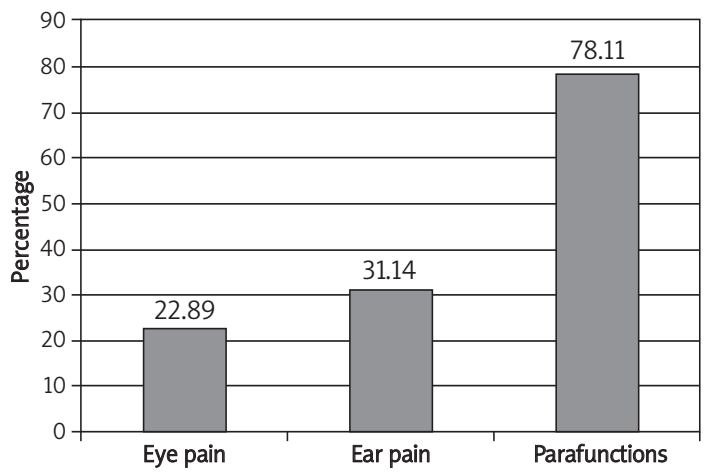

Figure 2. Average prevalence of symptoms of eye and ear and parafunctions among patients

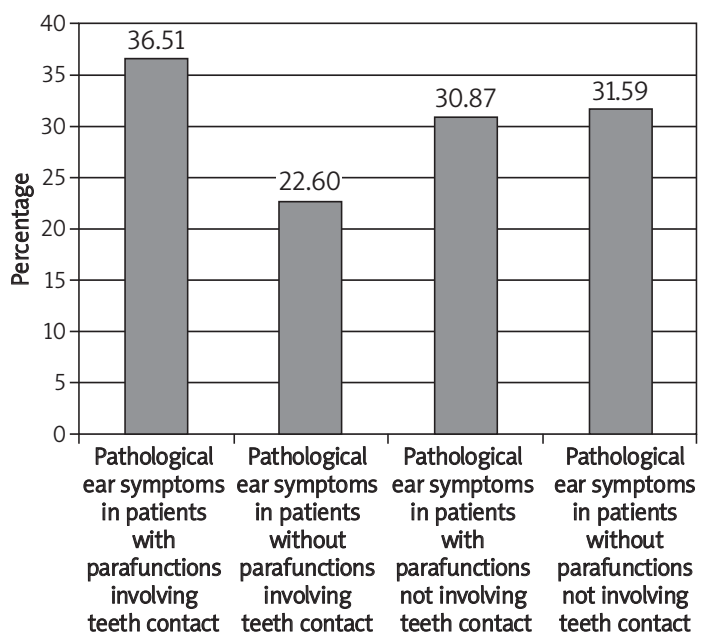

Figure 4. Effect of exercise teeth contact parafunctions and not teeth contact for symptoms of the ears 


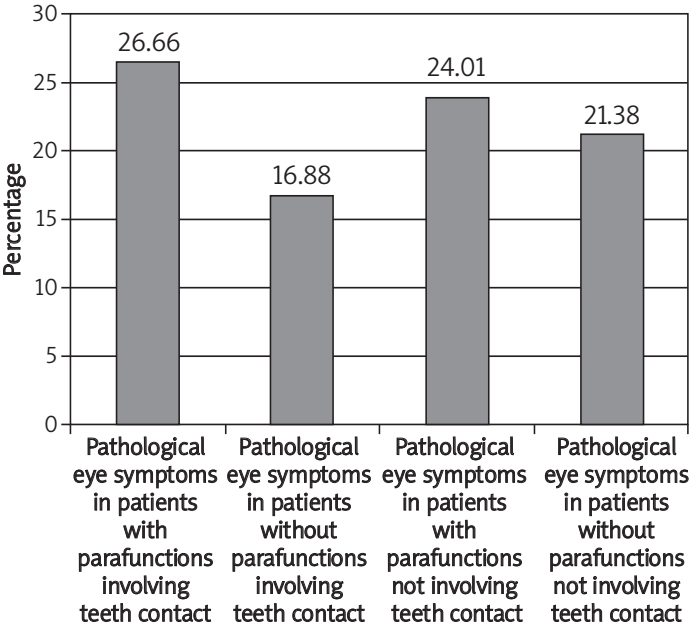

Figure 5. Effect of exercise teeth contact parafunctions and not teeth contact on the occurrence of eye symptoms

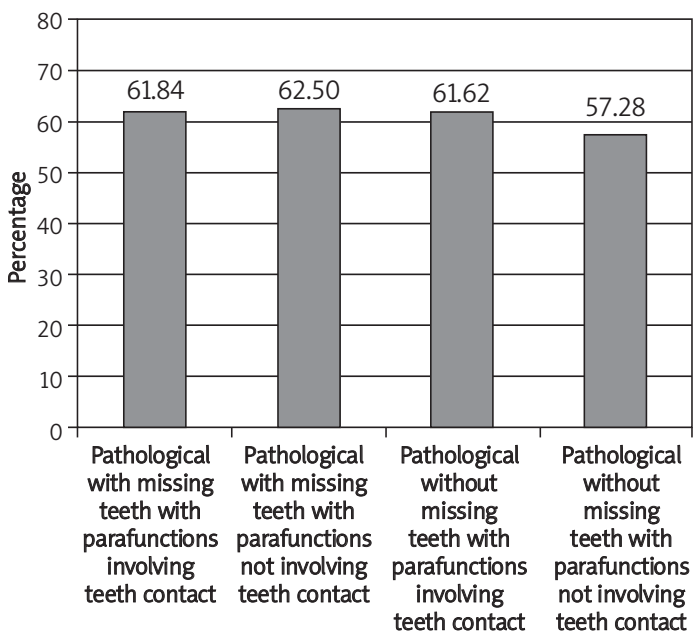

Figure 6. Percentage distribution of subjects carrying the teeth contact parafunctions with full teeth and tooth gaps identified, not teeth contact with full teeth and tooth gaps identified

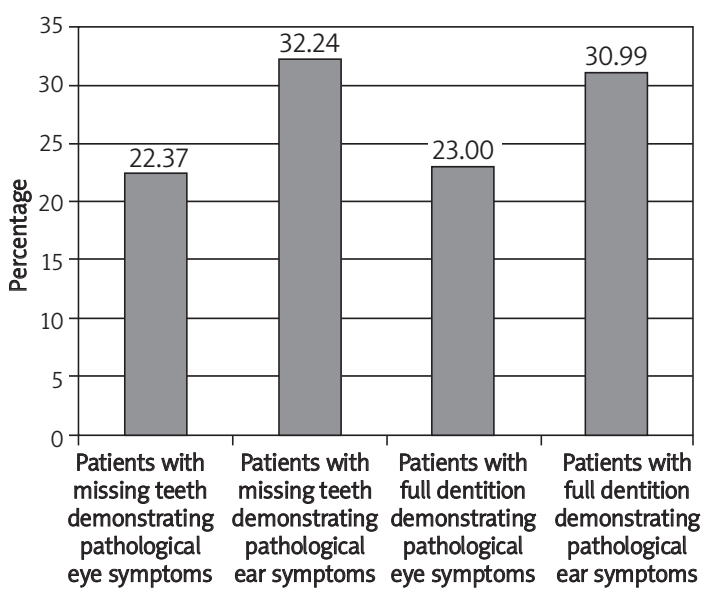

Figure 7. Occurrence of eye symptoms in patients with deficiencies and full dental arches and ear symptoms in patients with deficiencies and full dental arches ing teeth and full dentition who show signs of nontooth contact parafunctions) - the event is less likely to occur (Figure 6).

Taking into account the occurrence of eye and ear symptoms, they occurred in patients with missing teeth in $22.37 \%$ and $32.34 \%(n=152)$ and among those with full dentition among $23 \%$ and $30.99 \%$, respectively $(n=852)$. $\mathrm{RR}=0.97, \mathrm{Cl}=0.68$ 1.38, Pearson's $\chi^{2}$ : 029,569, df $=1, p=0.863472$ (for people with missing teeth and full dentition demonstrating pathological eye symptoms) - the event is less likely to occur. $\mathrm{RR}=1.05, \mathrm{Cl}=0.77-1.44$, Pearson's $\chi^{2}$ : 094,072, df $=1, p=0.759064$ (for people with missing teeth and full dentition demonstrating pathological ear symptoms) - the event is less likely to occur (Figure 7).

According to statistical data, the teeth contact parafunctions should be taken into account when diagnosing symptoms of eye and ear, whereas the non-teeth contact parafunctions seem not to play an important role in those disorders.

\section{Discussion}

The results of the research confirm that the described parafunctions tend to occur more and more often. Moreover, it appears that adolescents younger than 18 tend to seek professional help more frequently. It may be linked with the growing number of parafunctions induced by stress conditions, especially among young people [14-27]. As far as gender is concerned, women seem to seek advice and treatment more often at the Functional Disorders Laboratory at the Medical University of Lublin. This may be caused by the fact that women suffer from parafunctions more frequently, and the pain symptoms tend to be more explicit and more severe in this gender group $[8,9,11$, 13, 28].

During the development of the human organism, the relationship between the jaws changes. Until the age of 17 years the height of the face measured between the nasion and the gnathion points increases. Until the age of 30 years it stays the same, whereas afterwards the height of the lower face segment decreases as a result of tooth wear or tooth loss. The change in relationship between the maxilla and the mandible becomes one of the main causes of dysfunctions in patients in all age groups. Moreover, independently of age, pychoemotional factors contribute to the formation of parafunctions. These pychoemotional factors include personal and professional stress that becomes an inherent part of a parafunction. If a parafunction is left undiagnosed and untreated, it becomes habitual. Both missing teeth and fixed parafunctions influence the temporo-mandibular joints. They might manifest themselves as pathological abrasion of tuberculae and foveae or displace- 
ment of the articular disk. They are also the reason why patients seek professional help at the Functional Disorders Laboratory (FDL) (Figure 1) [3, 7-9].

The results of our findings confirm other studies describing the incidence of parafunctions. They occur in $80 \%$ of the 1006 people treated at the Department of Prosthodontics of the Medical University of Lublin in 2003-2008 (Figure 2). Nevertheless, one should not forget, according to the definition of a parafunction provided at the beginning of the article, that not each patient is able to recall the occurrence of any parafunction or may not be aware of its presence. It should be estimated that the rate at which those parafunctions occur is much higher. That is why parafunctions have become a very important factor in diagnosing diseases and disorders of the eye and ear. As shown in Figures 2 and 3 , they occur very often among the patients of the FDL. Moreover, the type of the parafunction (involving tooth contact or not involving tooth contact) is also very important. These first, as we have seen, should be important in diagnosing symptoms of ear and eye (Figures 4, 5). According to statistical data, the former should be taken into account when diagnosing symptoms of eye and ear, whereas the latter seem not to play an important role in those disorders (Figures 4, 5). It seems vital to determine the type of a parafunction because the kind of the harmful habit affects the occurrence of the eye and ear symptoms [2, 6, 9, 12, 29, 30].

It is important to combine parafunctions with symptoms of eye and ear the same as periodontal disease with heart disease [31, 32]. It is important that the new public health objectives have advice on how to protect patients from performing parafunctions in accordance with the recommendations of Kleinrok [4, 33, 34].

The frequency of occurrence of parafunctions among patients with missing teeth and full dentition is at the same level. Therefore, one could suppose that the smaller number of teeth does not affect the frequency of parafunctions very much. However, it may seem worth noting that the difference in the frequency of occurrence of parafunctions that do not involve tooth contact in patients with missing teeth is $5 \%(R R=1.09, \mathrm{Cl}=$ 0.95-1.25) (Figure 6). The occurrence of eye and ear symptoms in patients with missing teeth and full dentition is at the same level. Nevertheless, it cannot be assumed that the number of teeth does not affect the occurrence of eye and ear symptoms. The lack of support areas decreases the height of the bite and consequently it leads to changes in the temporomandibular joint (TMJ) that may result in either painful or painless symptoms $[4,9,15]$.

In conclusion, the highest ratio of missing teeth is among patients aged $30-60$ years, among whom tooth wear and tooth loss leading to a decrease in bite height may be observed. Because parafunctions are a frequent phenomenon, they should be taken into account when diagnosing diseases and disorders of the organs of vision and hearing. The kind of parafunction is very important. Only those involving tooth contact should be taken into consideration when diagnosing eye and ear syndromes. The data presented here show that the number of missing teeth does not have a significant influence on the frequency of occurrence of parafunctions.

\section{References}

1. Kleinrok M. Diagnostik Und Therapie von Okklusionsstorungen. Mit vielen praktischen Beispielen unter Verwendung des Funktiographen. Quintessenz Verlag. Berlin, Chicago, London, Sao Paulo, Tokio 1986.

2. Kleinrok M. Funcional disorders of the masticatory system [Polish]. Sanmedia, Warszawa 1992.

3. Koeck B, Graber G, Hupfauf L, et al. Funcional disorders of the masticatory system [Polish]. Maślanka T (ed.). Urban\&Partner, Wrocław 1997.

4. Kleinrok M. On the harmful effects and the need of treating masticatory system dysfunctions [Polish]. Zdrowie Publiczne 1991; 4: 152-8.

5. Krakowiak K, Kleinrok M, Mielnik-Hus J, DoraczyńskaBanach E. Habitual gum-chewing, dysfunctions of the masticatory system 1996; 46: 360-6.

6. Fleischer-Peters A, Scholz W. Psychologie und Psychosomatik in der Kieferorthopadie. Carl Hanser Verlag, Monachium 1985.

7. Kaczmarek A. Research into headache and its correlation to functional disorders of masticatory system [Polish]. AM, Lublin 1990.

8. Guichet NF. Clinical management of occlusally related orofacial pain and TMJ dysfunction. J Craniomand Pract 1983; 1: $60-73$.

9. Ingerslev $\mathrm{H}$. Functional disturbances of the masticatory system in school children. J Dent Child 1983; 50: 445-9.

10. Wigdorowicz-Makowerowa N. Zaburzenia czynnościowe narządu żucia [Polish]. PZWL, Warszawa 1984.

11. Kleinrok M. Painful and painless symptoms connected with dysfunction of the motor system of masticatory system [Polish]. Terapia 2004; 10: 19-27.

12. Kleinrok M. Research into correlation between dislocation of the articulatory disc and the position of the caput mandibulae [Polish]. Prot Stom 2000; 50: 71-9.

13. Kleinrok M, Kleinrok J. Dividing and recognising dislocation of the articulatory disc of the TMJ during during maximal cuspidation [Polish]. Prot Stom 2000; 50: 185-94.

14. Łata A, Karasiński A. Methods supporting the treatment of patients with TMJ and muscle dysfunctions - literature review. Part I Physiotherapy [Polish]. Mag Stom 2001; 11: 84-6.

15. Kato T, Thie NMR, Huynh N, Miyawaki S, Lavigne GJ. Topical review: sleep bruxism and the role of peripheral sensory influence. J Orofac Pain 2003; 17: 191-213.

16. Ciangaglini R, Gherlone EF, Radaelli G. The relationship of bruxism with craniofacial pain and symptomsfrom the masticatory system in the adult. J Oral Rehabil 2001; 28: 842-8.

17. Pergamalian A, Rudy TE, Zaki HS, Greco CM. The association between wear facets, bruxism and severity of facial pain in patients with temporomandibular disorders. J Prosthet Dent 2003; 90: 194-200. 
18. Castelo PM, Barbosa TS, Gaviao MB. Quality of life evaluation of children with sleep bruxism. BMC Oral Heath 2010; 14: 10-16.

19. Krzyżkowiak D, Krzyżkowiak W. Bruxism [Polish]. Post Psych Neurol 1994; 3: 519-21.

20. Panek $\mathrm{H}$. The rate of bruxism based on own indicator in patients with full natural dentition [Polish]. Prot Stom 2002; 52: 3-8.

21. Sawrasewicz-Rybak M, Ulatowski S, Split W. Musculo-fascial pain in the masticatory system. Part I. General characteristic of the syndrom [Polish]. Mag Stom 2006; 6: 22-4.

22. Ulatowski S, Marciniak S, Pawlak Ł, Sawrasewicz-Rybak M, Split W. Musculo-fascial pain in the masticatory system. Part III. Characeristics of trigger points in the masticatory muscles [Polish]. Mag Stom 2006; 7-8: 42-7.

23. Split M, Kowalski M, Pawlak Ł, Sawrasewicz-Rybak M, Suliborski B, Marciniak S. Musculo-fascial pain in the masticatory system. Part II. Treatment [Polish]. Mag Stom 2006; 9: 112-4.

24. Pawlak $九$, Marciniak S, Wierzbicka-Ferszt A, Split W. Musculo-fascial pain in the stomatognathic system [Polish]. Mag Stom 2008; 6: 20-5.

25. Kleinrok $M$. Stated needs and attempt of the prophylaxis of funcional disorders of the masticatory system [Polish]. Prot Stom 1989; 49: 177-83.

26. Kleinrok M. Pretreatment of the pain syndrom of the dysfuncion of the masticatory system [Polish]. Mag Stom 2002; 12: 12-6.

27. Litko M, Piórkowska-Skrabucha B, Czelej-Piszcz E, Kleinrok J. Masticatory system dysfuncions among patients under 18 based on the research of the Department of Functional disorders of the masticatory system, Medical University of Lublin [Polish]. Czas Stomatol 2007; 60: 119-27.

28. Gotębiewska M. The occurence of the symptoms of functional disorders among highschool students [Polish]. Prot Stom 1982; 32: 102-9.

29. Mielnik-Hus J, Żyśko-Woźniak D, Kleinrok M, Doraczyńska E, Szkutnik J, Kaczmarek A. Habitual nail-biting and dysfunctions of the masticatory system. Prot Stom 1994; 44: 200-4.

30. Żyśko-Woźniak D, Mielnik-Hus J, Kleinrok M, et al. Habit ual nail-biting and dysfunctions of the masticatory system. Prot Stom 1993; 43: 243-9.

31. Shanker J, Kakkar V. Role of periodontal infection in cardiovascular disease: a current perspective. Arch Med Sci 2009; 5: 125-34.

32. Jegier M, Smalc A, Ciesielski P, Jander S, Jegier B. Evaluation of the dental status in patients referred for heart surgery - a preliminary report. Arch Med Sci 2006; 2: 125-7.

33. Schaller B, Sandu N. Clinical medicine, public health and ecological health: a new basis for education and prevention? Arch Med Sci 2011; 7: 541-5.

34. White F, Nanan D. Community health case studies selected from developing and developed countries - common principles for moving from evidence to action. Arch Med Sci 2008; 4: 358-63. 